\title{
Improving concrete toughness by aggregate arrangement
}

\author{
Mija H. Hubler ${ }^{10 *}$, Shahlaa Al Wakeel ${ }^{2}$, Boning Wang ${ }^{1}$
}

${ }^{1}$ University of Colorado, Boulder, USA

${ }^{2}$ Saint Martin's University, Lacey, USA

${ }^{\circ}$ M.J. Hubler was invited for submission of this letter as an awardee of the Gustavo Colonnetti Medal granted by RILEM in 2020

Received: 20 May 2020 / Accepted: 03 August 2020 / Published online: 12 August 2020

(C) The Author(s) 2020. This article is published with open access and licensed under a Creative Commons Attribution 4.0 International License.

\begin{abstract}
The focus of this research is to address the following open question: Can we engineer the distribution of coarse aggregate in concrete to improve crack growth resistance? A finite element study of aggregates distributed in a notched 3-point bending concrete sample shows that the stress field in front of the crack tip and in the entire concrete beam is carried differently when the aggregate is arranged in a more orderly manner. Based on this, the impact of coarse aggregate arrangement is confirmed experimentally. Fracture toughness is determined from notched 3-point bending samples prepared from the same mix with three types of casting approaches: One following ASTM C31, and two following the standardized casting method with raking and shear mixing to arrange particles in a more ordered manner. To evaluate the impact of these added casting steps to alter aggregate arrangement, $\mathrm{X}$-ray computed tomography (XCT) data is collected of the particle positions for statistical analysis. XCT data shows that added raking and mixing during casting adjusts the arrangement of coarse aggregate with respect to each other in a systematic manner. The mechanical tests show increased toughness can be achieved when raking and shear mixing steps are added following the ASTM C31 casting process.
\end{abstract}

Keywords: Casting; Coarse aggregate; Fracture toughness

\section{Introduction}

There has been interest in crack control for improved resilience of concrete structures [1]. While cement mix composition has been studied and actively changed over the years, there has been much less research on the contribution of the coarse aggregate arrangement and on-site casting methods. Recent insights on the correlation between particle arrangement and the fracture toughness of composite materials have motivated the question if minor changes to aggregate arrangement in concrete could improve toughness of the material, increase the ability of a material to resist fracture without admixtures or any changes to cement chemistry.

Sun et al. [2] studied the effect of particle arrangement on stress concentrations in a composite material with a particle volume fraction of 0.3 . It was found that the stress concentration surrounding a particle is dominated by the nearest-neighbor particle and does not have a direct relationship with filler volume fraction. The composite consisted of BISGMA/TEGDMA polymer-matrix reinforced with E-glass-particles. Segurado et al.[3] studied the effect of particle spatial distribution on the stress fields in microstructures made up of $15 \%$ volume of spheres in a metal-matrix composite. It was found that the stress concentrations occurred in composite regions where the particles were closely packed. Previous theoretical and computational studies by Al Wakeel et al. [4] of glass spherical inclusions in a brittle polymer matrix revealed that toughness correlates to the degree of inclusion order. Huang et al. [5] numerically investigated the effect of coarse aggregate distribution in a two-phase composite material that consisted of cement mortar and coarse aggregate. The results of the analysis indicate that the concrete carbonation depth, which relates to cracking potential, decreases as the coarse aggregate distribution density increases. The experimental work done by Siregar et al. [6] investigated the effect of different aggregate size distributions on the fracture behavior of high strength concrete. Sixty-three notched beams were tested in 3-point bending using three aggregate size distributions and two water-to-binder ratios. The fracture toughness and energy were used to estimate the fracture characteristics of the concretes. The fracture toughness describes the material's ability to resist crack growth. The fracture energy is the energy required to open the crack surface by a unit area. It was found that the fracture energy

* Corresponding author: Mija H. Hubler, Email: hubler@colorado.edu 
decreases with increasing coarseness of aggregate. In contrast, reducing the total water-to-binder ratio increases the fracture toughness but has no effect on the fracture energy.

In this study finite element models are created to analyze how the arrangement of aggregates may affect the toughness of concrete. The aggregate arrangement is defined as the statistical relative spacing of the aggregate in a concrete section. Since the degree of order have been identified as a spatial metric that is correlated to the toughness of a composite [7], and it is something that can be globally adjusted, the aggregate arrangement is quantified using the degree of order based on the features of the radial distribution function. Once the differences in the stress field resulting from changes to the aggregate arrangement have been identified, changes to casting methods are considered to confirm the effect experimentally. Racking and shear mixing were selected as feasible changes to on-site concrete casting which may encourage an added fixed vertically spacing between aggregate or horizontally spacing between aggregate, respectively. While racking and mixing will encourage movement of coarse aggregate in various directions, and the material may slump back after these efforts, the work was conducted under the hypothesis that some characteristic spacing may remain upon hardening. Once the concrete has hardened XCT imaging is used to evaluate the effectiveness in introducing order, in the form of an additional characteristic length, in the aggregate arrangement due to these changes in casting. If the stress field is indeed changed by adjusting the coarse aggregate arrangement, a mechanical difference in the fracture toughness ought to be observed in concrete beams made with casting methods that introduce a more systematic spacing of the aggregate. This effect on the mechanical performance is also confirmed experimentally.

\section{Numerical Modeling}

Finite element models of notched 3-point bending tests of concrete beams are created with different arrangements of coarse aggregate. Two sets of six models each have random and slightly less random aggregate positions. After loading, the stress field is analyzed in the numerical model to evaluate the similarities and differences caused by the changes in coarse aggregate arrangement.

\subsection{Random Aggregate Distribution}

The open-source software toolbox Mote3D [8] can generate the randomly distributed positions of coarse aggregates with a specified size distribution within the sample domain. This software randomly positions spherical particles with defined minimum inter-particle distance in a cubical computational domain. To generate a distribution representative of coarse aggregate in concrete a mean particle diameter of $2 \mathrm{~cm}(0.8$ in.) is selected according to 26.4.2.1 (a)(5) of ACl 318-19 [9] with a standard deviation of $0.25 \mathrm{~cm}$ (0.1 in.). Kozul and Darwin concluded that, "coarse aggregate typically occupies over one-third of the volume of concrete" [10]. Therefore, for a cubic domain with an edge length of $12.7 \mathrm{~cm}$ (5 in.), 155 aggregate positions need to be generated for the selected aggregate size distribution. Particle placement which avoids overlap and a moderate packing density is achieved by running 50,000 trial particle positions. Mote3D uses a modification of the classical random sequential addition (RSA) scheme to generate particle positions along with neighbor distance reduction to increase packing (this reduction was intentionally disabled).

\subsection{Less Random Aggregate Distribution}

To generate a slightly less random distribution, aggregate positions are restricted to only allow generation in a grid of subdomains within the full computational domain. This way the random aggregate positions are generated, but their locations are loosely bounded in a systematic fashion. To assess the effectiveness of this method of introducing slight degree of order, the radial distribution function [11] of the aggregate positions is assessed for a cuboidal space containing a population of 602 particles. Figure 1 shows the effective emergence of peaks into the radial distribution function for the case of slightly less randomly distributed aggregate positions.
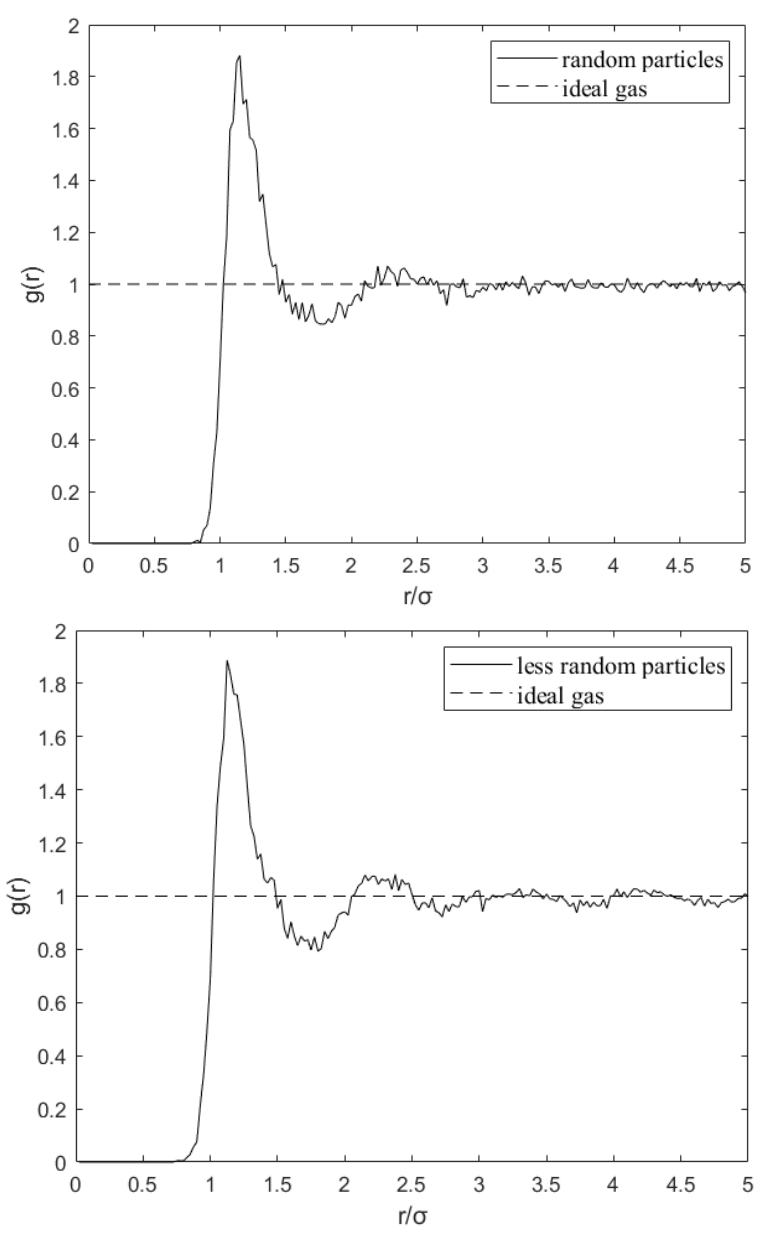

Figure 1. Radial distribution function of randomly distributed aggregate and aggregate particles which are random with spatial constraints. The random distribution with spatial constraints shows more prominent oscillations about 1 , the benchmark ideal gas molecule random arrangement, which illustrates the emergence of some characteristic spacings in the coarse aggregate arrangement. 


\subsection{Finite Element Model}

Python scripts are used to generate the solid distributed spheres in an Abaqus finite element model according to the identified random and less random aggregate distributions. The setup of the 3-point bending model is based on ASTM E399, and is shown in Figure 2. Imposing periodic boundary conditions on the aggregate arrangements, the beam is built by creating a $63.5 \times 12.7 \times 12.7 \mathrm{~cm}^{3}\left(25 \times 5 \times 5 \mathrm{in}^{3}\right)$ cuboid and copying the aggregates arrangements five times. The spheres are assigned coarse aggregate material properties, the surrounding material mortar, and the loading setup steel (See Table 1). The aggregate particles are embedded in the mortar paste. A straight through-cutting notch (according to ASTM E399) is placed at the center of the bottom edge of the beam. Semi-circular loading and support points are used to avoid stress concentration during loading. The beam is constrained to be in constant contact with the supports and loading bar. The left support is a pinned support, restricting movements in both the $x$ and $y$ direction. The right support is a roller support, restricting movements in the $y$ direction. Load is applied as a $689 \mathrm{kPa}$ (100 psi) pressure at the top bar. The particles are meshed with 10-node quadratic tetrahedron elements and the steel and mortar are meshed with 8-node linear brick elements. The material compliance of deformation of the beam and the supports is specified for each component. The materials were modeled as linear elastic without plastic or damage properties. A linear elastic finite element model enables the determination of the stress and strain fields around a pre-defined notch. The characteristics of the stress field in front of a crack directly impact the material's toughness and are studied in the numerical results.

Table 1. Material properties assigned in the model to provide a 34.5 $\mathrm{MPa}(5000 \mathrm{psi})$ strength concrete mix.

\begin{tabular}{|l|l|l|l|}
\hline & $\begin{array}{l}\text { Coarse } \\
\text { Aggregates }\end{array}$ & $\begin{array}{l}\text { A36 Steel } \\
{[14]}\end{array}$ & Mortar \\
\hline Density, kg/m $\mathrm{m}^{3}$ & $2700[18]$ & 7800 & $2200[15]$ \\
\hline Elastic modulus, GPa & $\begin{array}{l}68.95 \quad[13] \\
{[17]}\end{array}$ & 200 & $\begin{array}{l}20.68 \\
{[12][13][16]}\end{array}$ \\
\hline Poisson's ratio & $0.33[17]$ & 0.26 & $0.2[16][17]$ \\
\hline
\end{tabular}
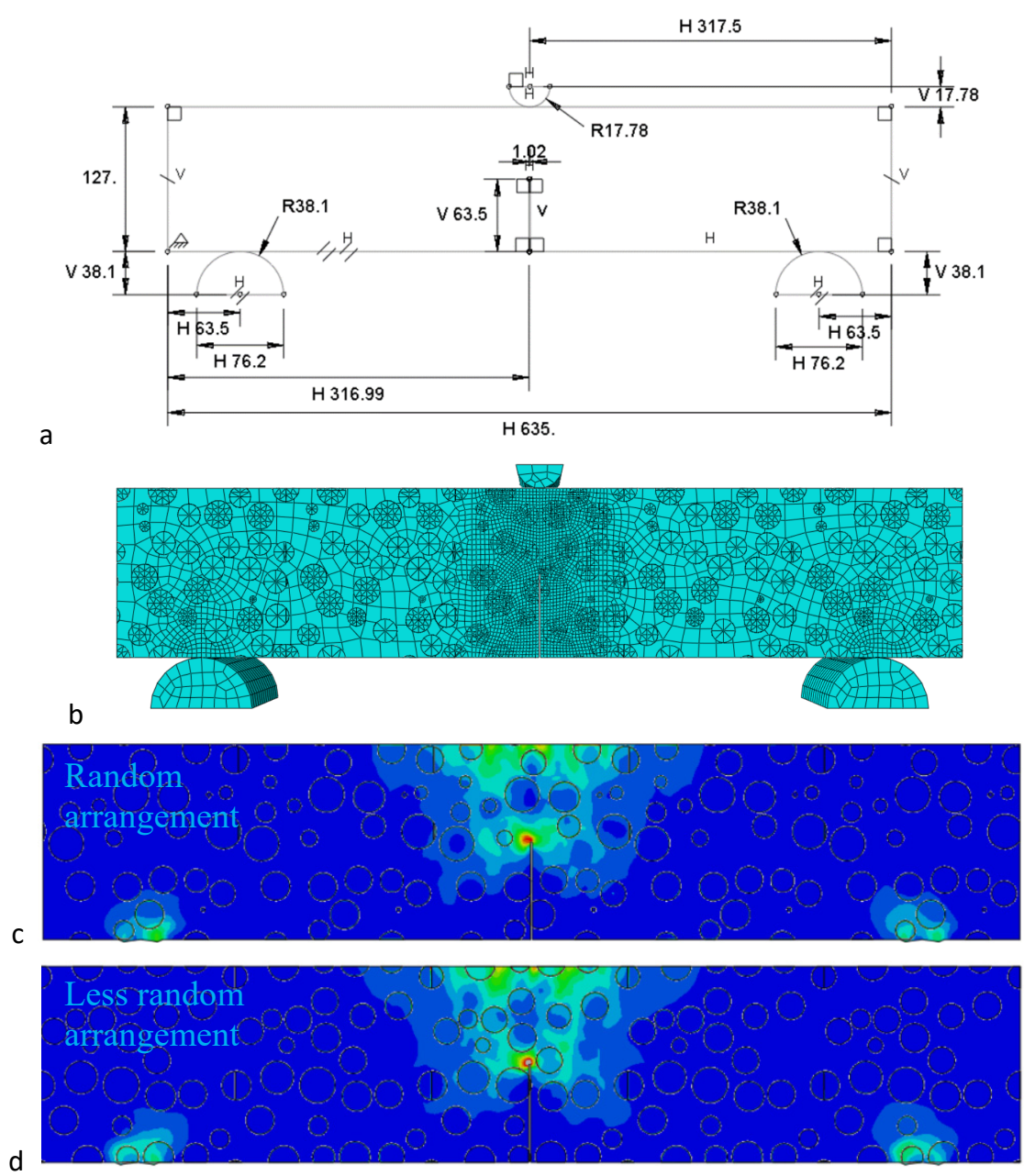

Figure 2. a) Dimensions ( $\mathrm{mm}$ ) of the numerical model and b) the mesh showing the embedded aggregate. Note that the combined hexahedron and tetrahedron mesh with 171000 nodes is refined near the crack to resolve the stress field. A typical Von Misses stress field for the random (c) and less random (d) aggregate distribution is shown. 


\section{$2.4 \quad$ Numerical Insights}

The resulting stress and strain distributions in the finite element model show the expected stress concentration at the crack tip with various points of minor stress localization throughout the aggregates based on the selected arrangement. The element-wise strain in the 6 models with randomly distributed aggregate and the 6 models with less randomly distributed aggregate were compared as a histogram. Figure 3 shows that the deviations between the two aggregate arrangements is mainly seen as differences in the number of low strain and stress elements across the entire beam. While high strain elements lead to higher stresses and drive failure, low non-zero strain elements are a sign of better stress distribution throughout the entire beam. More elements with low non-zero strains are seen to occur throughout the entire test specimen for a less random distribution of aggregate. This implies that slight ordering improves the global stress distribution but may increase the global sensitivity to flaws.
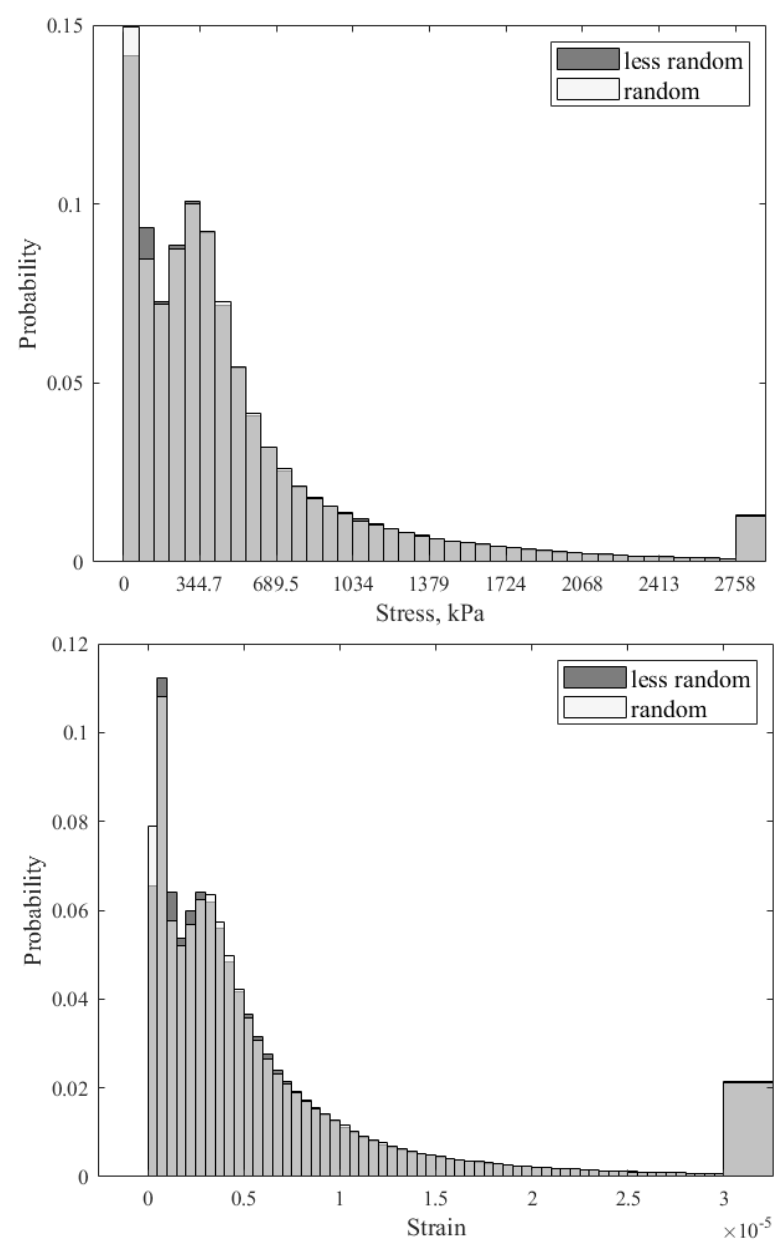

Figure 3. Combined histogram of element-wise strain and Von Misses stress magnitude for all 6 notched bending specimens with randomly arranged aggregate and all 6 notched bending specimens with less randomly arranged aggregate.

Features of the local stress field in front of the crack tip provide a visualization of the concrete's ability to distribute stress near a singularity. To systematically assess the differences in how the aggregate distributions impact the local stress field the magnitude of the stress field was plotted along a horizontal linear path just above the crack tip. The resulting stress measures, and their average, are shown in Figure 4. For less random aggregate arrangements the average stress in front of the crack tip shows a marginally smaller spread, with a higher peak, and is thus consistently engaging the material centered in front of the crack more. The 6 repeated realizations of the less random arrangement more frequently result in a higher stress in front of the crack, while the random arrangement shows more fluctuations in stress including some instances of lower stresses in front of the crack tip.

\section{Less Random Arrangement}

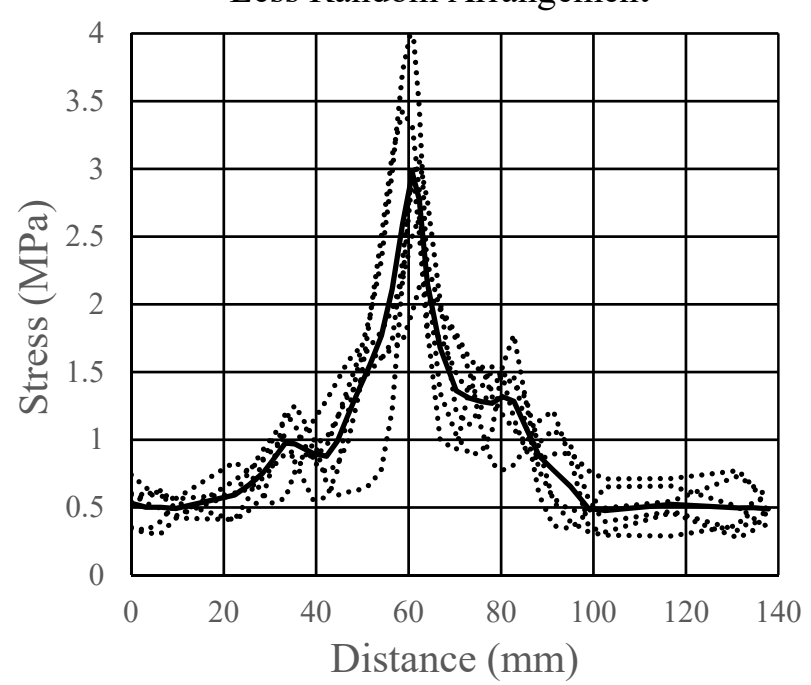

Random Arrangement

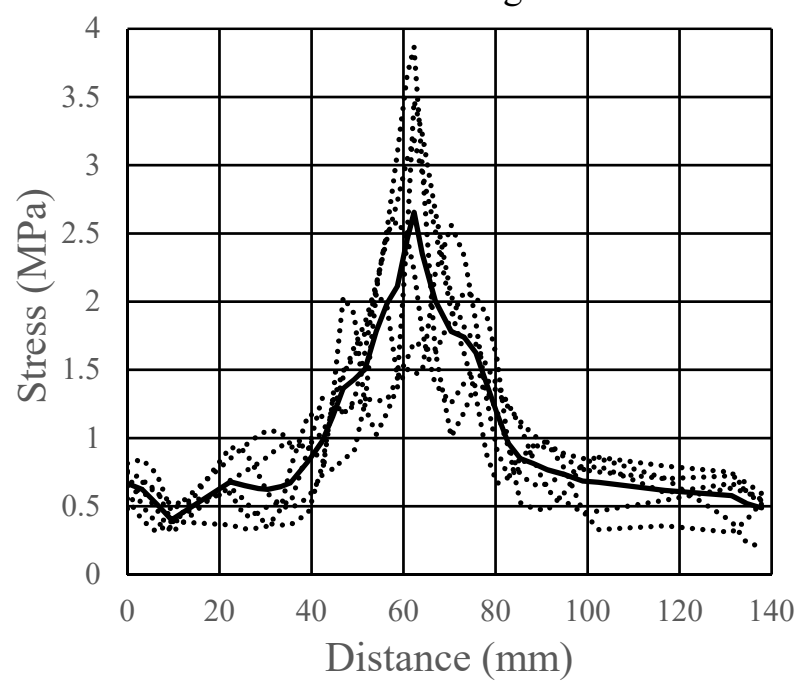

Figure 4. Horizontal linear stress path in front of the crack tip shown for each model in gray and averaged in black. The stress path is more concentrated along the crack path for the less random aggregate arrangement.

The finite element results reveal that there are differences in the strain and stress distributions in the concrete sample due to changes in the coarse aggregate distribution, although these are not visually discernable from the stress fields such as those shown in Figure 2. Considering the statistics of the 
strain and stress fields of many aggregate arrangements simultaneously we find that when aggregate is less random the entire concrete sample has a higher probability of exhibiting points of non-zero low strain (more nonlocal distribution of stress) and stresses are more likely to be higher near the crack tip.

\section{Experimental Investigation}

To confirm the effect of aggregate arrangement on the stress field observed in simulations, we conduct an experimental study which considers two methods of ordering aggregate in the mold during casting. For this different aggregate arrangements are generated using the same mix. To evaluate the effectiveness of the proposed changes to casting on coarse particle arrangement, an XCT scan is conducted and spatial statistics are evaluated of the coarse aggregate positions. Material toughness is used to assess the mechanical effect of the changing stress field since this directly relates to the material's ability to control cracking and is a common performance metric.

\subsection{Materials}

Using ACl 211.1-91 [19], a concrete mix is batched for six cylinders and 18 bending specimens. The composition is 340:228:840:872 kg/m $\mathrm{m}^{3}$ (cement: water: coarse aggregate: fine aggregate) or 21.23:14.23:52.44:54.44 lb/ $\mathrm{ft}^{3}$. By using a standard cone, the slump is found to be $76 \mathrm{~mm}$ ( $3 \mathrm{in}$ ). The coarse aggregate is sieved into a narrow range in size between $4.75 \mathrm{~mm}$ (0.187 in) and $9.5 \mathrm{~mm}$ (0.374 in).

\subsection{Test Specimens}

The specimen dimensions are selected to satisfy the ASTM E399 standard of plane strain requirements [20]. Based on the ASTM E399 standard, the notch is no more than $10 \%$ of the depth of the beam. The specimen's dimensions are: width $W$ equal to twice the thickness $W=2 B=100 \mathrm{~mm}(4 \mathrm{in})$, the crack length is half the width $\left(\frac{a}{w}=0.5 * 100=50 \mathrm{~mm}(2\right.$ in $\left.)\right)$, and specimen length $L=4 W=400 \mathrm{~mm}(16 \mathrm{in})$. The notch in each specimen is created by inserting a separator at the center of the specimen.

\subsection{Casting Methods}

Three different methods are used to generate different aggregate arrangements. The $1^{\text {st }}$ casting method followed ASTM C31 [21], and the $2^{\text {nd }}$ and $3^{\text {rd }}$ followed this method with a raking or mixing step, respectively. Concrete is placed in the mold in four layers, two below the notch and two above. Then, the faces of the mold are struck 15 times with a rubber mallet. A sketch of the raking and mixing added after this standard ASTM casting method is shown in Figure 5. For mixing a ' $T$ '-shaped shear mixer is attached to an electric drill and lowered halfway into the cast concrete. The wet mix is remixed at this elevation for less than a minute. For raking a two-prong fork is dragged through the wet concrete mix two times. All samples were covered with plastic sheeting and cured at room temperature for 28 days.
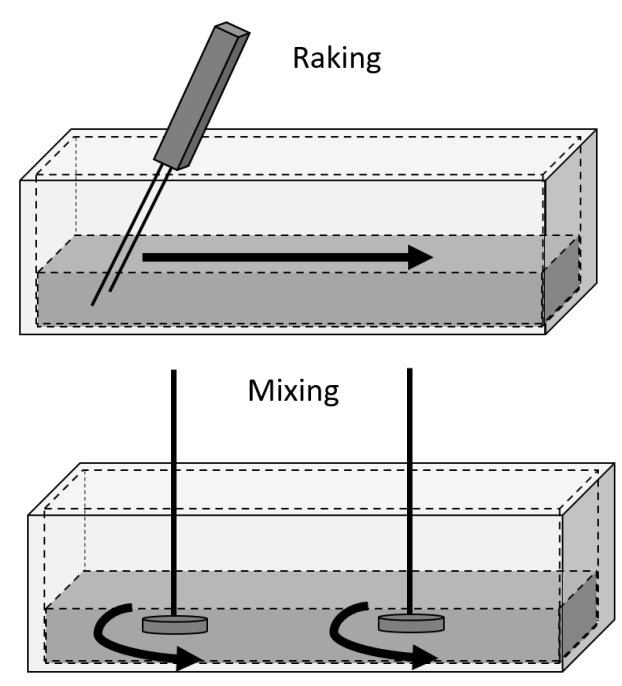

Figure 5. Illustration of the raking and shear mixing steps to introduce order in the casting process.

\subsection{Mechanical Testing}

The average cylindrical compressive strength of the concrete is $22 \mathrm{MPa}$. Six specimens are tested in 3-point bending for each casting method to determine the average fracture toughness. A constant displacement load is applied in a threepoint bending setup using an MTS compression testing machines. The notches are sharpened by running a new blade along the tip of the notch created by the separator during casting. The fracture toughness can be determined from the test data using the standard formula for the three-point bend geometry from the following equation:

$$
K_{I}=f\left(\frac{a}{w}\right) \frac{3 P S \sqrt{a}}{2 t w^{2}} \quad(\mathrm{MPa} \sqrt{\mathrm{mm}})
$$

where: $K_{I}$ is the stress intensity factor, $P$ is the load at failure, $S$ is the span length, $a$ is the crack length, $w$ is the specimen width, $t$ is the specimen thickness and $f\left(\frac{a}{w}\right)$ is a geometric function that can be calculated for this sample geometry using the equation below [22]:

$$
\begin{gathered}
f\left(\frac{a}{w}\right)=1.93-3.07\left(\frac{a}{w}\right)+14.53\left(\frac{a}{w}\right)^{2}-25.11\left(\frac{a}{w}\right)^{3}+ \\
25.80\left(\frac{a}{w}\right)^{4} \text { (dimensionless) }
\end{gathered}
$$

\subsection{XCT Imaging}

To characterize the internal aggregate arrangement, a Philips Gemini TF64 CT scanner is used to image the concrete macrostructure. Images are taken every $2 \mathrm{~mm}$ (0.08 in) through the sample with a $220 \mathrm{~mm}$ (8.66 in) field of view. The scanner has a spatial resolution of $0.75 \mathrm{~mm}(0.03 \mathrm{in})$ with a pixel spacing of $0.43 \mathrm{~mm}$ (1.69 in). This provides over 10 pixels across the typical aggregate to accurately identify the centroids of each aggregate. Three $200 \times 100 \times 50 \mathrm{~mm}^{3}(7.87$ $\times 3.94 \times 1.97 \mathrm{in}^{3}$ ) volumes are scanned from each casting method. 


\section{Experimental Results and Discussion 4.1 Toughness}

Figure 6 shows the results of the fracture toughness testing. For each casting method the average toughness and total spread of the test data is shown. ANOVA analysis confirmed that each casting group of 6 samples showed statistically significant differences in the mean value. Compared with the ASTM standard casting method, mixing provides a $6.5 \%$ increase in toughness and raking results in a $14.2 \%$ increase.

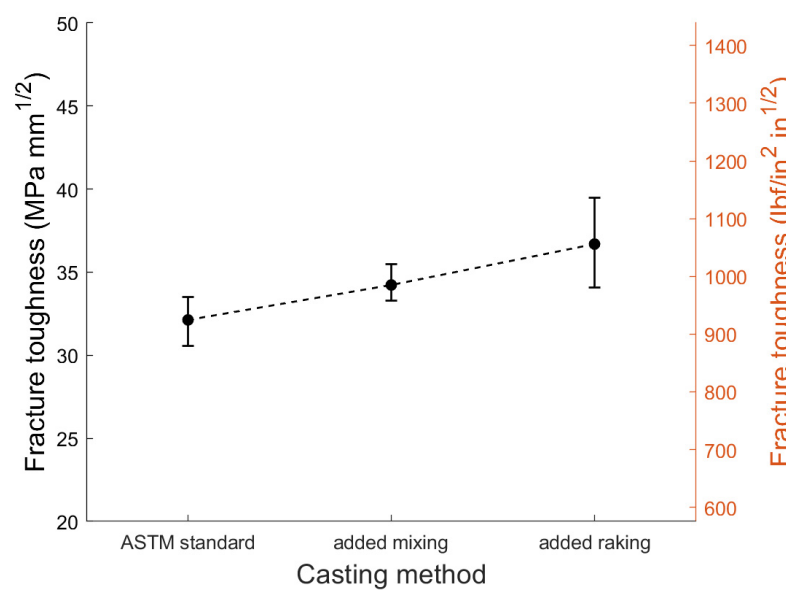

Figure 6. Experimental fracture toughness of concrete beams cast using ASTM standard methods, followed by mixing or raking. The data points indicate the mean and the whiskers show the standard deviation of each sample set.

\subsection{XCT Imaging and Aggregate Arrangement Statistics}

Figure 7 shows sample sections taken from the image reconstructions. The figures feature $\mathrm{X}$-ray shadows due to the heterogeneous material properties of the coarse aggregate within the cement matrix. Visual inspections did not detect differences in the distributions of the aggregates as a result of the different casting methods. As a result, statistical analysis of the coarse aggregate arrangement was performed.
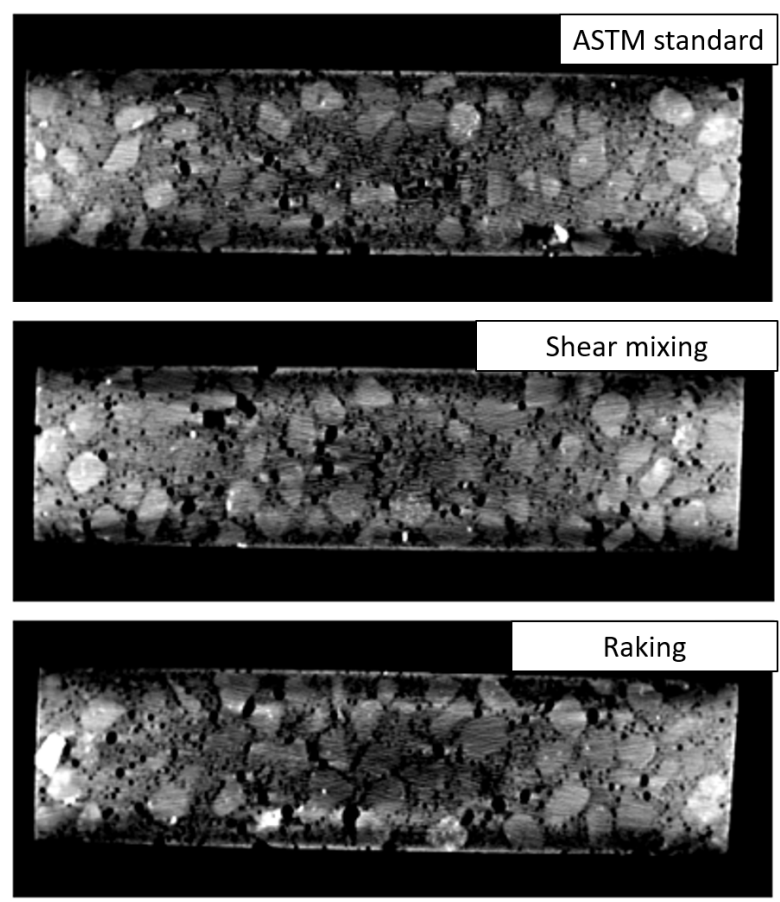

Figure 7. Characteristic image slices from XCT scans of the samples taken from each type of casting method.

Aggregate centroid positions were extracted by assuming approximately circular and/or oval cross-sections of aggregates, they were marked in each imaged slice, and confirmed by replication of the selection process. The aggregates were identified as circular or oval based on limits set for the relative sizes the axes of an ellipse matching the region of similar gray-scale intensity. Images were spaced closely such that each aggregate appears in multiple scans. This allowed regions that were identified as an aggregate in any image to be confirmed with similar regions in adjacent scans. On average 6,300 aggregate centroid positions were identified for each casting method. Each set of scans captured a total imaged volume, $V$. The set of $N$ aggregate positions in this volume were analyzed using a radial distribution function normalized by the number density of the system multiplied by the volume of the spherical shell. The relative radial distances from each aggregate centroid to all other aggregate centroids, were binned by radial distances, $d r$, and normalized by $\rho 4 \pi r^{2} d r$. The results are plotted in Figure 8. 

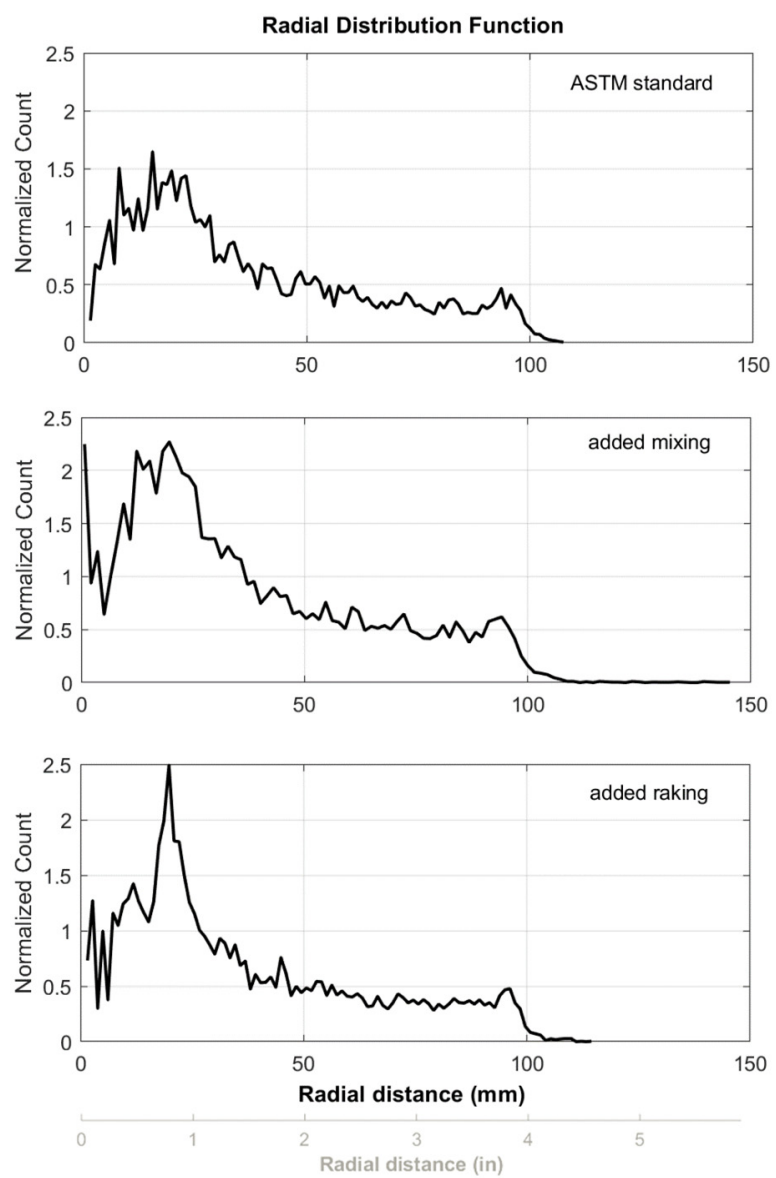

Figure 8. Radial distribution statistics of the coarse aggregate identified in the 3 XCT scanned volumes for each casting method. On average 6,300 aggregate centroid positions in 3D space were used to generate each distribution.

Radial distribution statistics represent the transition from random to ordered spatial arrangement [7]. Peaks in the radial distribution function capture the emergence of characteristic spacings in the $3 \mathrm{D}$ arrangement. The radial distribution of the coarse aggregates in the 3 casting methods is similar in shape beyond $50 \mathrm{~mm}$ (1.97 in) but shows the emergence of short-range order when raked or mixed. A peak around $20 \mathrm{~mm}$ ( $0.79 \mathrm{in})$ increases in prominence from 1.5 to 2 with added mixing and becomes even more pronounced (reaching 2.5) with added raking. This size is just less than the spacing of the rake prongs.

\subsection{Relation Between Arrangement and Toughness}

The peaks observed in Figure 8 confirm that the added casting steps that were implemented had a lasting impact on the aggregate arrangement and introduced a characteristic spacing between the aggregate centroids, as hypothesized. The fracture toughness in Figure 6 shows a positive correlation to the short-range order in the aggregate arrangement. Raking with two passes of a 2-prong rake had a bigger impact on both arrangement and toughness, than the mixing approach.

\subsection{Expected Impact on Strength Probability Distribution}

The probability distribution and size effect scaling of strength of structures are mechanically linked to the characteristic size when quasibrittle materials are used. For concrete the characteristic size is correlated to the aggregate size [22]. While we are not changing the aggregate size in this work, the additional steps to casting is introducing a secondary characteristic size with the newly established spacing between aggregate that has not been previously considered. This $2^{\text {nd }}$ characteristic size could impact the form of the strength distribution, such as the tail, which dictates the reliability-based performance of concrete structures. Work by $\mathrm{Xu} \&$ Le [23] investigated the connection between length scales in a nonlocal strength-based failure model for the strength distribution of quasibrittle materials, and the basic material properties. They were able to explicitly consider both material strength and applied stress field, as well as the statistical correlation features of the material. They conclude that while the statistical correlation found in the material does impact the mean strength (as shown by this work), it does not impact the form of the strength distribution. Based on these insights, it is expected that the changes to casting methods proposed here will not have a detrimental impact on the reliability-based design of concrete.

\section{Conclusions}

Finite element models of concrete beams reveal that aggregate arrangement changes the global stress field in the bending sample and local stress field in front of a crack. The experimental study reproducing this effect showed that raking after casting is more effective at ordering aggregate and improving concrete fracture toughness. Since the material can locally slump back after each pass of the fork tool, it is anticipated there will be a bigger impact with more passes of the tool. A key insight gained from this work is that spatial arrangement of coarse aggregate may be treated with the theories of statistical physics to improve concrete toughness.

The results of this study imply that coarse aggregate arrangement may be used as a design variable to control crack development. Considering how concrete will fracture is crucial during structural design to achieve safety [24], based on this work it should also be considered during mix design and casting to improve structure durability. Both the spatial arrangement of the coarse aggregate and the cement composition play a role in the material's resistance to crack development. In lieu of or in addition to changes in cement composition, it may be economical to consider minor alterations to casting methods to improve concrete durability. Future work focused on optimizing this method for different concrete mixes and beam sizes could be done to establish a standard casting process for regions of structures prone to excessive cracking. 


\section{Contributions}

Each author contributed equally to the study. Wang conducted all numerical investigations and their analyses. Al Wakeel conducted all experimental investigations and their analyses. Hubler directed the study, processed and analyzed the XCT data, and prepared the manuscript.

\section{References}

[1] P.K. Mehta, R.W. Burrows, Building durable structures in the 21st century. Indian Concrete Journal (2001) 75(7): 437-443.

[2] C.J. Sun, P. Saffari, K. Sadeghipour, G. Baran, Effects of particle arrangement on stress concentrations in composites. Mater Sci Eng J (2005) 405: 287-295. https://doi.org/10.1016/j.msea.2005.06.032

[3] J. Segurado, C. Gonzalez, J. Lorca, A numerical investigation of the effect of particle clustering on the mechanical properties of composites. Acta Materialia J (2003) 5: 2355-2369. https://doi.org/10.1016/\$1359-6454(03)00043-0

[4] S. Al Wakeel, F. Ghanbari, M. Hubler, Particle arrangement effects on the stress intensity in composite material. Eng Fract Mech J (2018) 202: 33-46. https://doi.org/10.1016/j.engfracmech.2018.09.014

[5] Q. Huang, Z. Jiang, W. Zhang, X. Gu, X. Dou, Numerical analysis of the effect of coarse aggregate distribution on concrete carbonation. Constr Build Mater (2012) 37: 27-35.

https://doi.org/10.1016/j.conbuildmat.2012.06.074

[6] A.P.N. Siregar, M.I. Rafiq, M. Mulheron, Experimental investigation of the effects of aggregate size distribution on the fracture behavior of high strength concrete. Constr Build Mater (2017) 150: 252-259. https://doi.org/10.1016/j.conbuildmat.2017.05.142

[7] S. Torquato, Random heterogeneous materials: microstructure and macroscopic properties. Springer-Verlag New York, Inc. 2002, 59-70.

[8] H. Richter. Mote3D: an open-source toolbox for modelling periodic random particulate microstructures. Model Simul Mater Sci Eng (2017) 25(3): 035011. https://doi.org/10.1088/1361-651X/aa629a

[9] American Concrete Institute (ACl) Committee 318. Building Code Requirements for Structural Concrete (ACl 318-14), 2019.

[10] R. Kozul, D. Darwin, Effects of Aggregate Type, Size, and Content on Concrete Strength and Fracture Energy. University of Kansas Center for Research, Inc., 1997.

[11] J.M. Haile, Molecular Dynamics Simulation. Chapter 6: Section 6.4: Static Structure. John Wiley \& Sons, New York, 1992.

[12] G. Rigby, R.N. Swamy, Dynamic properties of hardened paste mortar and concrete. Mater Struct (1971) 4: 13-40. https://doi.org/10.1007/BF02473927

[13] A. Neville, Properties of Concrete (Fifth Edition). Chapter 3: Properties of aggregates, Chapter 9: Elasticity, shrinkage, and creep. 2012.

[14] MatWeb. ASTM A36 Steel, plate. Retrieved April 2020.

[15] W. Jackiewicz-Rek, K. Zalegowski, A. Garbacz, B. Bissonnette. Properties of Cement Mortars Modified with Ceramic Waste Fillers. Procedia Engineering (2015) 108: 681-687. https://doi.org/10.1016/i.proeng.2015.06.199

[16] H. Djayaprabha, T. Chang, J. Shih, Comparison Study of Dynamic Elastic Moduli of Cement Mortar and No-cement Slag Based Cementitious Mortar Activated with Calcined Dolomite with Impulse Excitation Technique. MATEC Web of Conferences (2018) 186: 02004 https://doi.org/10.1051/matecconf/201818602004

[17] R.N. Swamy, Dynamic Poisson's Ratio of Portland Cement Paste, Mortar and Concrete. Cem Concr Res (1971) 1: 559-583. https://doi.org/10.1016/0008-8846(71)90060-3

[18] M. Kosmatka, S. Wilson, Design and Control of Concrete Mixtures, Chapter 5: Aggregates for Concrete. Portland Cement Association. 2016.

[19] ACl 211.1-91. The American Concrete Institute. Standard practice for selecting proportions for normal, heavyweight, and mass Concrete. 2002.

[20] ASTM E399. Standard test method for Linear-Elastic Plain-Strain Fracture Toughness KIC of Metallic Materials. 2012, New York.

[21] ASTM C31. Standard Practice for Making and Curing Concrete Test Specimens in the Field. 2014, New York.

[22] Z.P. Bažant, J. Planas, Fracture and size effect in concrete and other quasibrittle materials, CRC press, (1997.

[23] ACl 318-14. The American Concrete Institute. Building Code Requirements for Structural Concrete, 2014.
[24] Z. Xu, Z., \& Le, J. L. (2018). On power-law tail distribution of strength statistics of brittle and quasibrittle structures. Engineering Fracture Mechanics, 197, 80-91. https://doi.org/10.1016/j.engfracmech.2018.04.009 